\title{
Experience of Hydatid Cyst Management in a Tertiary Care Center in Kathmandu Nepal
}

\author{
Bijendra D Joshi, Udaya Koirala, Arbin Joshi, Saroj P Dhital, Prativa Dhoubadhel \\ Department of Surgery, Kathmandu Model Hospital, Kathmandu, Nepal
}

Corresponding author:

Bijendra D Joshi, MBBS, MS

Department of Surgery, Kathmandu Model Hospital, Kathmandu, Nepal

Email: bijendra441@hotmail.com

Submitted : Feb 25, 2020

Accepted : Apr 5, 2020

\begin{abstract}
\section{Introduction}

Hydatid cyst is a common disease in Asian subcontinent produced by the larval stage of Echinococcus granulosus. The most common site of involvement is the liver. The mode of presentation can vary. The management of hydatid cyst includes medical treatment with albendazole, ultrasound guided aspiration and instillation of scolicidal agents as well as surgical treatment. Surgery can be perfomed by open or laparoscopic approach. The aim of this study is to analyze the cases of hydatid cyst of liver managed in our set up.
\end{abstract}

\section{Methods}

This is a retrospective study of 45 patients with hydatid cyst managed in Kathmandu Model Hospital over the last five years. Data were collected from the patients hospital records. Demographic parameters, chief complains, radiological findings, anti echinococcal immunglobulin findings, mode of management,complications of surgery were evaluated.

\section{Results}

Majority of our patients were female (66.7\%). Mean age of the patients were $39 \pm 17.2$ years (16 to 89 years).Most common symptom of presentation was pain in right upper quadrant of abdomen.Most common site of involvement was liver (93.33\%)followed by spleen (4.4\%). There was one case of ruptured pulmonary hydatid cyst which was referred.Operative management was done in patients $(17.77 \%)$ out of which five patients (62.5\%) underwent laparoscopic partial pericystectomy and omentoplasty.No major complications were encountered in any of the cases managed surgically.

\section{Conclusion}

Majority of cases occured in liver. Most cases were managed conservatively. Laparoscopic management of hydatid cyst is effective.

Keywords: Hydatid cyst, laparoscopic surgery, liver 


\section{INTRODUCTION}

E chinococcal (Hydatid) disease has been known from the time of Hippocrates, who described patients with liver full of water. ${ }^{1,2}$ In Nepal the burden of hydatid cyst is quite significant but minimal work has been done to study the impact. The commonest organ affected is the liver $(75.21 \%)$ followed by lung $(14.5 \%){ }^{2}$ Diagnosis is done with clinical features, imaging findings and immunoglobulin assay. The management of hydatid cyst includes medical treatment with albendazole along with surgical treatment. More than $80 \%$ of liver hydatid cyst have favorable outcome without pre-emptive surgical intervention and can be efficiently managed with long term albendazole. [3]Laparoscopic pericystectomy is the most appropriate surgical treatment. ${ }^{4,5}$

\section{METHODS}

This is a retrospective study conducted at the Department of Surgery, Kathmandu Model Hospital. Chart review of patients with hydatid cyst who presented to the hospital between November 2012 to November 2017 was done. Approval was taken from the Institutional Review Committee (IRC) at the hospital. Data were retrieved from the patients' hospital records. Forty five patients with hydatid cyst were diagnosed during this period but four patients were lost to follow up and four patients opted for treatment at other centers..

The data gathered were mode of presentation (right upper quadrant pain, jaundice, fever, vomiting, abdominal lump or accidental findings), clinical examination findings, imaging (ultrasound and computed tomography abdomen and pelvis, chest $X$-ray) findings, immunoglobulin test (IgG for Echinococcusgranulosus) and management approach.

The diagnosis was done on the basis of history, clinical examination, imaging and immunoglobulin test. Ultrasonography was screening imaging followed by contrast enhanced computed tomography as per the WHO informal working group (IWGE) guidelines. 9, 10,13,16,17

All patients were treated with three cycles of four weeks albendazole tablets $400 \mathrm{mg}$ twice daily with two weeks of drug free interval in between the cycle. Further management plan was decided according to size of the cyst.

If the cyst size was less than $5 \mathrm{~cm}$ one more cycle of albendazole was given and follow up scan was done after one month. If the cyst size was between 5-10 cm patient was kept on follow up at 3-6 months intervals. Surgery was done in cysts larger than 10 $\mathrm{cm}$ and in patients who did not respond to medical treatment.

All surgeries were performed under general anaesthesia via laparoscopic approach. Patients who were not feasible with laparoscopic approach were converted to open surgery. Patients were followed up and any post operative complications were noted.

\section{RESULTS}

Forty five patients were diagnosed with hydatid cyst but four patients were lost to follow up and four were operated at other centers. Among them 30 patients were female $(66.7 \%)$ and 15 were male $(33.3 \%)$. The mean age was $39.87 \pm 17.2$ years (1689) years.

Right upper quadrant pain was present in $35(77.8 \%)$, jaundice was in $2(4.4 \%)$, fever in $2(4.4 \%)$, and vomiting in $2(4.4 \%)$ patients respectively. Incidental diagnosis of hydatid cyst was done in $3(6.7 \%)$ patients. One $(2.3 \%)$ patient was diagnosed while undergoing imaging for other problem. The serum

Table 1. Profile of patients $(n=45)$

\begin{tabular}{lc}
\hline \multicolumn{1}{c}{ Characteristics } & Number \\
\hline Gender & \\
Female & $30(66.7 \%)$ \\
Male & $15(33.3 \%)$ \\
Mean age & $39.87 \pm 17.2$ \\
Clinical presentation & \\
Right upper quadrant pain & $35(77.8 \%)$ \\
Jaundice & $2(4.4 \%)$ \\
Fever & $2(4.4 \%)$ \\
Incidental & $3(6.7 \%)$ \\
Vomiting & $2(4.4 \%)$ \\
Abdominal lump & $1(2.3 \%)$ \\
Location & \\
Liver & $42(93.3 \%)$ \\
Spleen & $2(4.4 \%)$ \\
Lung & $1(2.2 \%)$ \\
Size of cyst & \\
$<5$ cm & $19(42.2 \%)$ \\
$5-10$ cm & $10(22.3 \%)$ \\
$>10$ cm & $16(35.5 \%)$ \\
WHO classification of cyst* & \\
CE1 & $31(68.9 \%)$ \\
CE2 & $6(13.3 \%)$ \\
CE3 & $5(11.1 \%)$ \\
CE4 & $2(4.45 \%)$ \\
CE5 & $1(2.3 \%)$ \\
${ }^{*}$ CE: cystic echinococcosis & \\
CE1: unilocular, anechoic , double line sign \\
CE2: multiseptate honey comb cyst \\
CE3: cyst with detached membrane, daughter cyst \\
CE4: heterogenoushypoechoic/hyperechoic content/ \\
no daughter cyst \\
CE5: solid calcified wall \\
\end{tabular}


Table 2. Summary of management $(n=37)^{*}$

\begin{tabular}{lc}
\hline \multicolumn{1}{c}{ Management } & Number \\
\hline Treatment modality & $3(6.66 \%)$ \\
Open cystectomy & $5(11.1 \%)$ \\
Laparoscopic pericystectomy & $1(2.2 \%)$ \\
Referred to thoracic surgeon & $28(62.28 \%)$ \\
Conservatively managed & \\
Surgical management based on size & $16(62.5 \%)$ \\
$>10 \mathrm{~cm}$ & $10(20 \%)$ \\
$5-10 \mathrm{~cm}$ & - \\
$<5 \mathrm{~cm} *$ & 3 \\
Complications & 0 \\
Open surgery** & \\
Laparoscopic surgery & \\
$*$ Out of 45 patients, 4 patients lost in follow-up, 4 \\
patients opted for treatment in other centers \\
* Managed medically \\
** Wound infection-1, Fever-1, Chest infection-1
\end{tabular}

antibody (lgG) by ELISA was positive in 21 (46,6\%) patients.

The most common site for the hydatid cyst was liver in 42 (93.3\%) followed by spleen in 2 (4.4\%) and lung in $1(2.2 \%)$ patients respectively (Table 1$)$.

Cyst size less than $5 \mathrm{~cm}$ in $19(42.2 \%)$ patients, 5-10 $\mathrm{cm}$ in $10(22.3 \%)$ patients and more than $10 \mathrm{~cm}$ in 16(35.5\%)patients. The mean cyst size was $7.14 \mathrm{~cm}$ (Table 1).

According to WHO classification $81 \%$ of cysts were active [CE1(68.9\%) and CE2 (13.3\%)], transitional cyst (CE3) in (11.1\%), and inactive in $7 \%$ of patients [CE4(4.45) and CE5 were (2.3\%)] respectively (Table 1).

All patients were treated with two cycles of four

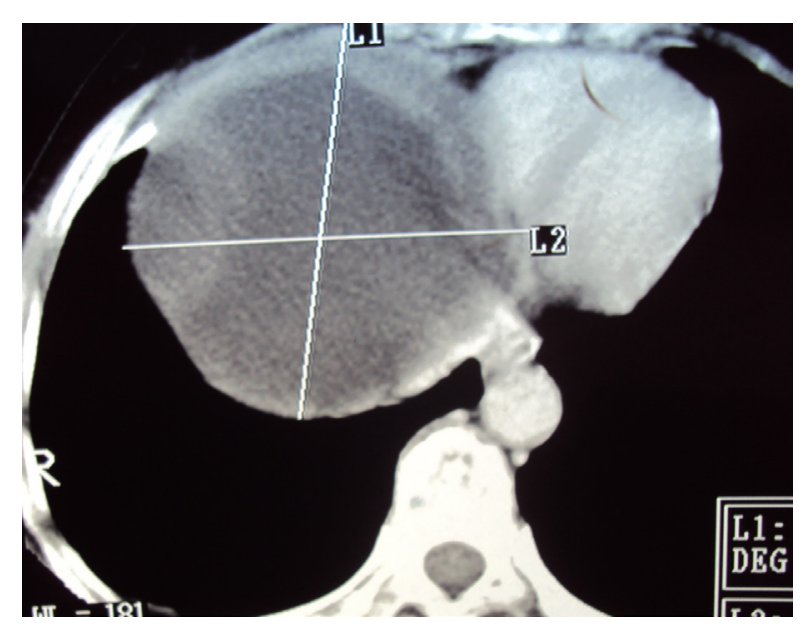

Fig 1. Computed tomography of abdomen showing a hypodense lesion involving the right lobe of liver, likely hydatid cyst weeks albendazole (10 mg/kg/day in divided doses) $400 \mathrm{mg}$ twice daily with two week of drug free interval in between the cycles as recommended by WHO. Twenty eight patients(62.28\%) were treated with albendazole therapy. Among them 19 patients had cyst size less than $5 \mathrm{~cm}$. Nine patients showed decrease in cyst size with inactive cyst at follow up. Operative management was done in 8 patients $(17.77 \%)$ out of which 5 patients $(62.5 \%)$ underwent laparoscopic partial pericystectomy and omentoplasty and 3 patients $(6.66 \%)$. One case of ruptured pulmonary hydatid cyst was referred to the thoracic surgeon (Table 2). The computed tomography (CT) image of abdomen showed hypodense lesion in right lobe of liver likely hydatid cyst (Figure 1). Intraoperatively hydatid cyst with daughter cysts were identified (Figure 2).

Four patients were lost to follow up and four patients were operated in other hospitals (Table 2).

No major complications were noted in cases managed with laparascopic pericystectomy with omentoplasty. Average median follow up was 7 months ranging from 1-21 months.

\section{DISCUSSION}

Hydatid cyst is an infestation caused by the larvae of Echinococcusgranulosus.Man is an accidental intermediate host in the evolution of this parasite. This disease has created public and environment problems in all areas of Nepal. In spite of public health concern, very little study has been done in Nepal for its treatment modalities. ${ }^{1}$

The commonest organ affected is the liver $(75.21 \%)$ followed by lung (14.5\%). ${ }^{2}$ More than $80 \%$ of liver hydatid cyst have favorable outcome without preemptive surgical intervention and can be efficiently managed with long term albendazole. ${ }^{3}$ In some

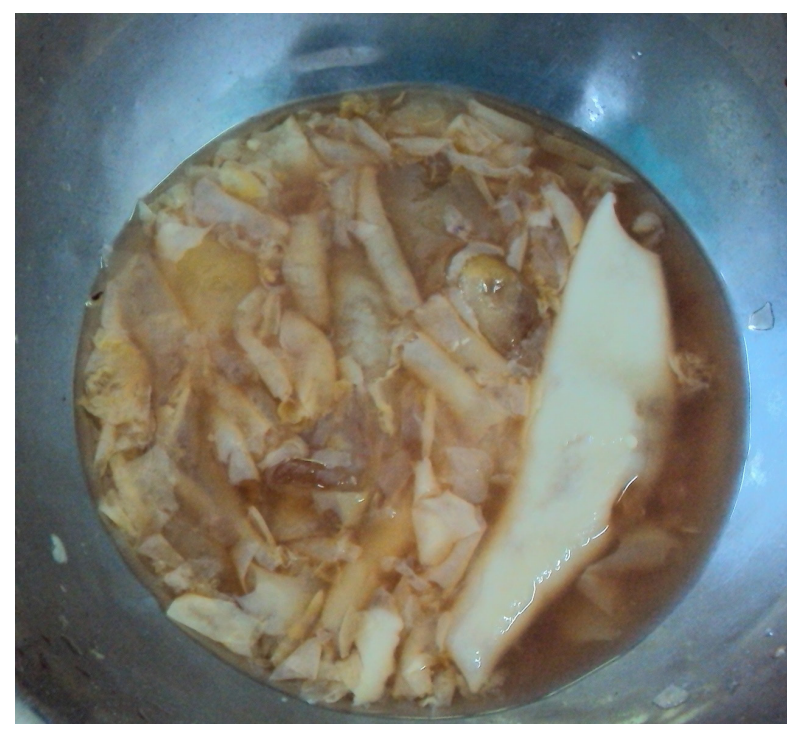

Fig 2. Hydatid cyst membrane and daughter cysts 
studies it is shown that surgical treatment with pre and post-operative administration of albendazole is the best treatment options for liver hydatid cysts due to low recurrence and complications rate. ${ }^{4}$

In this study majority of the patients were female (66.7\%). Many studies (Rao SS et. al, Kayal A et. al, Shrestha Sk et. al.) have reported female predominance whereas some has reported male predominance. $2,5,6$

Most of the patients presented with right upper quadrant pain (77.8\%), followed by jaundice $(4.4 \%)$, fever $(4.4 \%)$ and vomiting $(4.4 \%)$ which has also been reported by Shrestha et. al., Pradhan et. al. These studies were also conducted in Nepal. 6,7,8,19 Right upper quadrant pain and jaundice were the most common findings in these studies as well.

In our study 28 (62.2\%) patients treated with albendazole therapy showed decrease in cyst size at follow up in 9 patients with $<5 \mathrm{~cm}$ cyst. In a similar study of 32 patients put on albendazole for 8 weeks at the dose of $10 \mathrm{mg} / \mathrm{kg} / \mathrm{day}$ no recurrences were seenat follow up for 12 weeks $(p<0.01)$ with only $9.37 \%$ viable cysts at the time of surgery as compared to $96.87 \%$ who did not receivealbendazole therapy. ${ }^{15}$ In another study of 64 patients with albendazole therapy only $9.37 \%$ had viable cyst at the time of surgery as compared to $96.87 \%$ of patients who did not received any preoperative albendazole therapy. The recurrence rate was less than $4.16 \%$ in patients who received albendazole therapy compared to recurrence rate of $18.75 \%$ in patients who did not receivealbemdazole therapy. ${ }^{24}$

Laparoscopic pericystectomy with omentoplasty was the standard surgical treatement at our center. Laparoscopic pericystectomy has been performed by various investigators. 6,7,8,18,20 The techniques applied at open surgery can all be performed laparoscopically and include puncture and aspiration, deroofing and drainage, deroofing and omentoplasty,partial and complete pericystectomy. [18]Tuxon et al. reviewed the world literature on laparoscopic treatment of liver hydatid cyst. ${ }^{21}$ They reported on data from 57 published articles with a total of 914 patients. Eighty nine percent were completed laparoscopically and 5\% treated with open cystectomy. ${ }^{21}$ In two studies done by Shrestha $\mathrm{S} K$ et al and Ghimire P.S et al in tertiary centre the results were similar. Out of 26 patients, 24 patients underwent laparoscopic pericystectomy (92.3\%), 2 patients were convered to open surgery (7.69\%).6,7 In another single institution study of 10 year period, out of 62 patients,59 (95.1\%) underwent laparoscopic surgery and 3 (4.84\%) required conversion to open surgery. ${ }^{22}$ In a similar study involving surgical management of 30 patients, 24 (80\%) underwent complete pericystectomy and 4 (13.4\%) underwent partial pericystectomy. ${ }^{23}$ In a study done by Mousavi SR et al,omentoplasty of the cyst cavity was preferred compared to tube drainage. ${ }^{25}$

No significant complications were noted in patients treated with laparoscopic pericystectomy, while wound infection, fever and chest infection were noted in patients with open surgery. In other studies also minimum morbidity and no mortality were noted. ${ }^{19,20}$

\section{CONCLUSION}

Majority of cases occured in liver. Most cases of hydatid cyst of liver can be managed with medical therapy alone. Operative treatment both open and surgical is a developing field in our centre.

\section{CONFLICT OF INTEREST}

None declared.

\section{REFERENCES}

1. Joshi DD, Joshi AB. Epidemiology of echinococcus in Nepal. Southeast Ascon J Trop Med Public Health:1997;28 Suppl1:26-31.

2. Rao SS, Mehra B,Narang R.The spectrum of hydatid disease in rural central India. An 11Year experience .Ann Trop Med Public Health 2012;5:225-230

3. Saidi $\mathrm{F}$ et al, The Non-Operative Management of Asymptomatic Liver Hydatids; Ending Echinococcophobia. J Gastrointest Surg,2017

4. Gomez C et al, Review of the treatment of liver hydatid cyst. World JGastroenterol 2015,7;21(1),121-131.

5. Kayal A, Hussain A.A complete perspective clinical study of hydatiddisease.ISRMGastroenterology. vol 2014,Article ID514575

6. Shrestha SK,Thapa P et al.Laparoscopic Approach for management of hydatid cyst liver. J Nepal health Res Counc 2017 Jan-Apr;15(35):67-70.

7. Pradhan S, Ghimire B, Kanskar P et al. Laparoscopic management of hydatid cyst of liver. Journal of Institute of Medicine;Aug-Dec,2016,38:2-3,37-41

8. Darbari A, Jauhari A, Shrivasta A, Management of hepatic hydatidosis by open versus laparoscopic surgery. Int J Sci,2014 Nov;2(4):1371-1377

9. Wuestenberg J,Beate $\mathrm{G}$ et al.:Diagnostics in cystic echinococcosis:Serology versus ultrasonography. Turk J Gastroenterol 2014;25:398-40

10. Marrone G, Grino F et al. Multidisciplinary imaging of liver hydatidosis; World J Gastroenterol 2012;18(13):1438-1447

11. Park SJ,Han SS et al. Prevalence of serum IgG antibodies to cystic echinococcus antigen among patients in Uzbekistan Emergency Hospital. Korean J Parasitol 2015 Dec;53(6):699-703

12. Adjjadi SM, Sedaghat F et al. Serum Antigen and 
Antibody Detection in Echinococcosis: Application in Serodiagnosis of Human Hydatidosis. Korean J parasitol2009 Jun,vol47,N2:153-157

13. Kumar MJ, Toe K, Banerjee RD; Hydatid cyst of liver. Postgrad Med J 2003;79:113-114.

14. Guidelines for treatment of cystic and alveolar echinococcus in humans. Bull World Health Organ. 1996:74(3):231-242

15. Ul-Bari S, Arif SH et al.Role of Albendazole in the Management of Hydatid Cyst Liver. Saudi J Gastroenterol,2011 Sep-Oct;17(5):343-347

16. Stojkovic $M$, Rosenberger $\mathrm{K}$ et al. Diagnosing and Staging of cystic Echinococcosis: How do CT and MRI perform in comparison to Ultrasound. PLOS Hegl Trop Dis.2012 oct;6(10):e1880

17. WHO Informal Working Group;International Classification of Ultrasound images in Cystic Echinococcosis for application in clinical and field epidemiological settings.Acta Tropica vol 85,Issue 2,Feb 2003;253-261

18. Anand S, Rajagopalan S,Mohan R. Management of liver hydatid cysts-Current Perspectives.Medical journal Armed forces India 68(2012):304-309

19. Maquim R, Kamran et al.Laparoscopic treatment of Hepatic HydatidCyst.Journal of the college of Physicians and Surgeons Pakistan:2011, vol 21(8),468-471

20. Yagmur $Y$, Akbulut $S$ et al. Laparoscopic management of hydatid cyst of liver.S.Afr J Surg 2016:54(3),14-17

21. Tuxun T, Zhang JH et al.World review of laparoscopic treatment of liver cystic echinococcosis-914 patients. Int J Infect Dis.2014 Jul;24:43-50

22. Zaharie $F$, Bartos D et al. Open or laparoscopic treatment of hydatid disease of the liver? A 10 year single institution experience.Surg Endosc2013 Jun;27(6):2110-2116

23. Gattu EM, Chopada M. Hydatid cyst of liver: different modalities og management and outcome, Int.Surg.J2017Jan;4(1)220-223

24. Arif SH, Bari SA et al. Albendazole as an adjuvant to the standard surgical management of hydatid cyst of liver.International journal of Surgery;6(2008):448-451

25. Mousavi SR, Khoshnevis J ,Kharazm P. Surgical treatment of hydatid cyst of liver:Drainage versus omentoplasty.Annalsn of Hepatology;4(4)2005:272-274 